Research Article

\title{
The Protective Effect of Cordycepin on D-Galactosamine/ Lipopolysaccharide-Induced Acute Liver Injury
}

\author{
Jin Li, ${ }^{1}$ Liping Zhong, ${ }^{2}$ Haibo Zhu, ${ }^{3}$ and Fengzhong Wang ${ }^{1}$ \\ ${ }^{1}$ Institute of Food Science and Technology, Chinese Academy of Agricultural Sciences (CAAS), Beijing 100193, China \\ ${ }^{2}$ Life Science College of Tarim University, Xinjiang 843300, China \\ ${ }^{3}$ State Key Laboratory for Bioactive Substances and Functions of Natural Medicines, Beijing Key Laboratory of New Drug \\ Mechanisms and Pharmacological Evaluation Study, Institute of Materia Medica, Chinese Academy of Medical Sciences \& Peking \\ Union Medical College, Beijing 100050, China
}

Correspondence should be addressed to Haibo Zhu; 392938376@qq.com and Fengzhong Wang; wangfengzhong@sina.com

Received 7 September 2016; Revised 3 February 2017; Accepted 5 February 2017; Published 28 March 2017

Academic Editor: Anshu Agrawal

Copyright (C) 2017 Jin Li et al. This is an open access article distributed under the Creative Commons Attribution License, which permits unrestricted use, distribution, and reproduction in any medium, provided the original work is properly cited.

\begin{abstract}
As the major active ingredient of Cordyceps militaris, cordycepin (3'-deoxyadenosine) has been well documented to alleviate inflammation and oxidative stress both in vitro and in vivo. To explore the potential protective effect of cordycepin in fulminant hepatic failure, mice were pretreated with cordycepin for 3 weeks followed by D-galactosamine (GalN)/lipopolysaccharide (LPS) injection. Then we found cordycepin $(200 \mathrm{mg} / \mathrm{kg})$ administration elevated survival rate, improved liver function, and suppressed hepatocyte apoptosis and necrosis in mice with severe hepatic damage by GalN/LPS treatment. Further, cordycepin inhibited hepatic neutrophil and macrophage infiltration and prevented proinflammatory cytokine production possibly through suppressing TLR4 and NF- $\kappa \mathrm{B}$ signaling transduction. The blockade of reactive oxygen species (ROS) and lipid peroxidation production by cordycepin was associated with the decrease of NAD(P)H oxidase (NOX) activity. Besides, cordycepin significantly prevented excessive autophagy induced by GalN/LPS in the liver. These data suggested that cordycepin could be a promising therapeutic agent for GalN/LPS-induced hepatotoxicity.
\end{abstract}

\section{Introduction}

Fulminant hepatic failure is an unusual but dreaded disease with high mortality, characterized by hepatic dysfunction, coagulation disorder, and later multiple organ failure. D-galactosamine (GalN) and lipopolysaccharide- (LPS-) induced acute liver injury is one of the most commonly used murine model resembling hepatitis virus invasion. LPS is considered as the primary inducer to liver injury, while GalN deteriorates the extent of hepatocyte necrosis by inhibiting RNA and protein synthesis [1]. Recent pharmacological researches demonstrated that LPS treatment combined with GalN promotes proinflammatory cytokine generation in hepatic macrophages, including interleukin (IL)-6, IL- $1 \beta$, and tumor necrosis factor- $\alpha$ (TNF- $\alpha$ ), which triggers caspase cascade and bring death to hepatocytes [2]. Concretely, TNF- $\alpha$ binds to its receptor (TNFR-1), recruiting and cleaving caspase- 8 through Fas-associated protein with death domain (FADD). Activated caspase- 8 truncates BH3-only BID protein and thus causes cytochrome $\mathrm{c}$ released from mitochondria, cleaving and activating caspase-9. Both caspase- 8 and caspase- 9 contribute to caspase-3 activation and finally result in cell apoptosis [3]. Subsequently, activated macrophages and neutrophils produce massive reactive oxygen species (ROS) through $\mathrm{NAD}(\mathrm{P}) \mathrm{H}$ oxidase (NOX), which accelerates hepatocyte necrosis in further [4].

Cordycepin, also known as 3'-deoxyadenosine, is one of the main active constituents of the traditional herbal medicine Cordyceps militaris, with a broad spectrum of biological activities, including initially identified antiviral, antifungal, and antitumorigenic activities [5-7]. It was later found that cordycepin possesses anti-inflammatory, antioxidative, antithrombotic, and antiadipogenetic activity as well [8-11]. In LPS-stimulated macrophages, pretreatment with cordycepin suppressed TNF- $\alpha$ expression and stimulated the production 
of anti-inflammatory cytokines IL-10 and NO $[12,13]$. In LPS-induced human epithelial cells, cordycepin effectively decreased vascular adhesion molecule-1 (VCAM-1) expression and prevented neutrophil infiltration [14]. In high-fat diet or alcohol-induced hepatotoxicity, cordycepin exerted beneficial effects on damaged livers [15, 16]. However, whether cordycepin can prevent acute liver injury caused by GalN/LPS remains unknown. In the present study, we investigated the protective effects and preliminary molecular mechanism of cordycepin on GalN/LPS-induced fulminant hepatic failure. Specifically, cordycepin prolonged survival time, improved liver function, and inhibited hepatocyte apoptosis and necrosis. Notably, cordycepin alleviated inflammatory damage in the liver via suppressing TLR4 expression, inactivating $\mathrm{NF} \kappa \mathrm{B}$ pathway, and thus decreasing circulating TNF- $\alpha$ level. It attenuated oxidative injury possibly by reducing NOX activity and inhibiting ROS production. Besides, we also found that cordycepin could decrease excessive levels of autolysosomes in the liver induced by GalN/LPS challenge.

\section{Materials and Methods}

2.1. Treatment of Animals. Male C57Bl/6J mice (18-22 g) were purchased from Charles River Corporation (Beijing, China), given access to food and water ad libitum. All the experiments confirmed to the guidelines from the Animal Care and Committee of the Institute of Materia Medica, Chinese Academy of Medical Sciences \& Peking Union Medical College (Beijing, China). Mice were given oral gavage of cordycepin (50 and $200 \mathrm{mg} / \mathrm{kg}$; purity $>98 \%$; Boyle Chemical Corporation, Shanghai, China) dissolved in $0.5 \%$ carboxyl methylcellulose sodium salt (CMC, vehicle) daily for consecutive 3 weeks. The corresponding control mice were administrated with an equivalent volume of vehicle. On the last day, the mice were injected intraperitoneally with GalN (700 mg/kg; J\&K Scientific, Beijing, China) and LPS $(15 \mu \mathrm{g} / \mathrm{kg}$; Escherichia coli, O111:B4; Sigma-Aldrich, St. Louis, USA) dissolved in phosphate-buffered saline (PBS) buffer at $1 \mathrm{~h}$ after cordycepin or vehicle administration. For survival experiment, forty mice were randomly assigned into the following groups (each group, $n=10$ ): (a) $200 \mathrm{mg} / \mathrm{kg}$ cordycepin-treated control group, (b) vehicletreated GalN/LPS group, (c) $50 \mathrm{mg} / \mathrm{kg}$ cordycepin-treated GalN/LPS group, and (d) $200 \mathrm{mg} / \mathrm{kg}$ cordycepin-treated GalN/LPS group. The survival rate of each group was monitored for $24 \mathrm{~h}$. For biochemical analysis, forty mice were randomly divided into four experimental groups (each group, $n=10$ ): (a) vehicle-treated control group, (b) $200 \mathrm{mg} / \mathrm{kg}$ cordycepin-treated control group, (c) vehicletreated GalN/LPS group, and (d) $200 \mathrm{mg} / \mathrm{kg}$ cordycepintreated GalN/LPS group. Blood and liver samples were harvested at $5 \mathrm{~h}$ after GalN/LPS injection.

2.2. Analysis of Liver Enzymes and Serum Cytokine Levels. The blood samples were drawn from the orbit, and serum were separated after two hours at room temperature. Alanine aminotransferase (ALT) and aspartate aminotransferase (AST) activities were analyzed using commercial kits from
Nanjing Jiancheng Bioengineering Institute (Nanjing, China). Circulating levels of cytokines TNF- $\alpha$, IL-6, IL-1 $\beta$, and necrotic marker high mobility group box 1 (HMGB1) were quantified using enzyme-linked immunosorbent assay (ELISA) kits according to the manufacturer's instructions (Cloud-Clone Corporation; Houston, USA).

2.3. Analysis of Histopathology. The liver samples were frozen in OCT embedding medium, and $6 \mu \mathrm{m}$ thick sections were prepared. Then the slices were stained with hematoxylineosin, and the histological changes were evaluated in nonconsecutive, randomly chosen histological fields. Necrotic areas were given in [\%] of liver area (five sections in each group) [17]. Apoptotic cells were detected by the terminal deoxynucleotidyl transferase-mediated dUTP nick-labeling (TUNEL) method using an in situ apoptosis detection kit (TaKaRa Co., Shiga, Japan). The number of TUNEL-positive cells was recorded. At least three tissue sections in each group were analyzed.

2.4. Immunohistochemistry. After treatment with hydrogen peroxide, frozen sections were incubated with rabbit anti-F4/80 antibody (diluted at $1: 200$, catalogue number: sc-26643, Santa Cruz Biotechnology, Dallas, USA) for $1 \mathrm{~h}$ at $37^{\circ} \mathrm{C}$. The sections were then incubated in secondary antibody following three washes (catalogue number: SAP-9100, Sino Biological, Beijing, China). Prior to counterstaining with hematoxylin, these sections were colored using diaminobenzidine (DAB) kit. Negative controls were established using rabbit IgG instead of primary antibodies. At least three tissue sections in each group were analyzed.

2.5. Analysis of Hepatic Lipid Peroxidation and NOX Activity. Liver tissues were homogenized in a saline buffer to prepare a 1/10 homogenate. The levels of malondialdehyde (MDA), glutathione (GSH), myeloperoxidase (MPO) activity, and NOX activity were measured using detection kits according to the manufacturer's instructions (Nanjing Jiancheng Bioengineering Institute, Nanjing, China). Final results were normalized by the total protein concentration of indicated samples.

2.6. Reactive Oxygen Species (ROS) Assay. The hepatic ROS content was determined as described previously [18]. Briefly, freshly prepared liver homogenate was incubated with $2^{\prime}, 7^{\prime}$ dichlorofluorescin diacetate (DCFH-DA) (Sigma-Aldrich, St. Louis, USA) at a final concentration of $10 \mu \mathrm{mol} / \mathrm{L}$ for $15 \mathrm{~min}$ at $37^{\circ} \mathrm{C}$. After centrifugation, the pellet was resuspended in potassium phosphate buffer $(\mathrm{pH} 7.4)$ and incubated for $60 \mathrm{~min}$ at $37^{\circ} \mathrm{C}$. Fluorescence was measured at wavelengths of $488 \mathrm{~nm}$ for excitation and $525 \mathrm{~nm}$ for emission. The amount of $2^{\prime}, 7^{\prime}$-dichlorofluorescein (DCF) was corresponded to a standard DCF curve. Final results were normalized by the total protein concentration of indicated samples.

Frozen liver sections were stained with dihydroethidium (DHE, Ex: $\mathrm{Em}=510 / 595 \mathrm{~nm}$ ) and observed under a fluorescence microscope (BX51W1; Olympus, Japan). All images were obtained by Volocity software (6.3; PerkinElmer, 

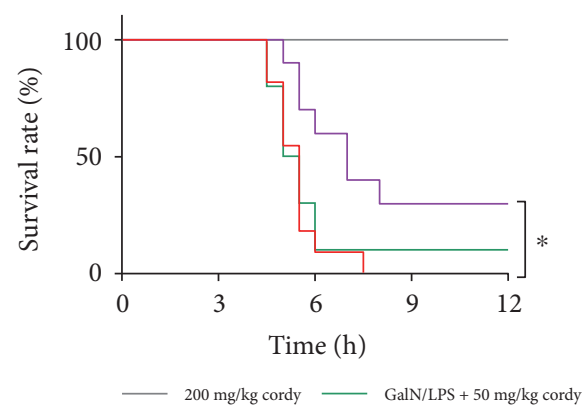

GalN/LPS _ GalN/LPS $+200 \mathrm{mg} / \mathrm{kg}$ cordy

(a)

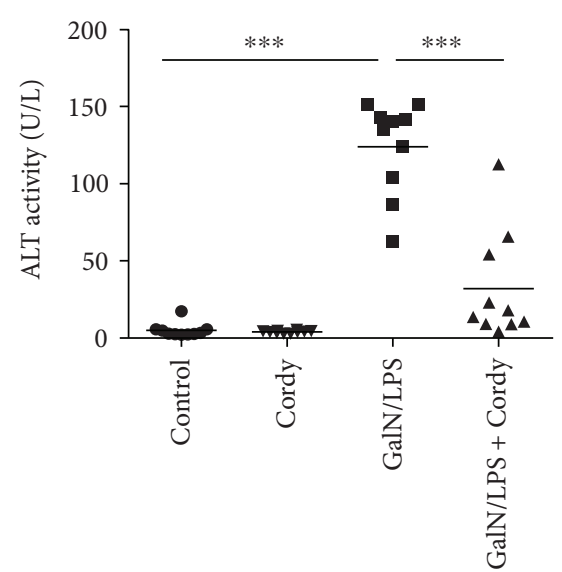

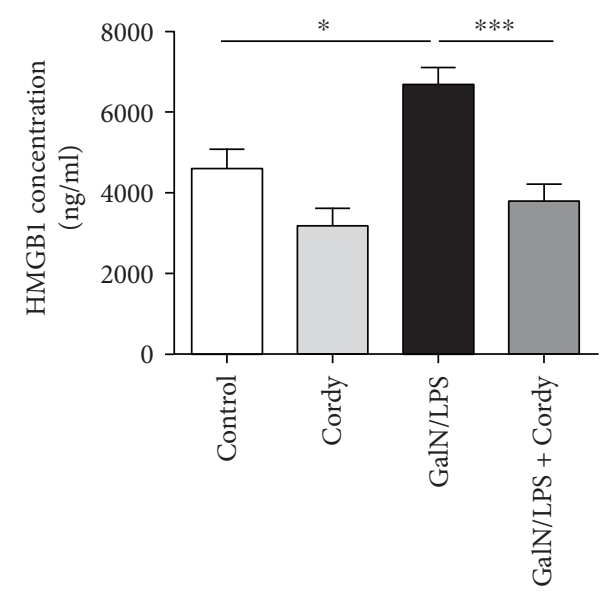

(b)

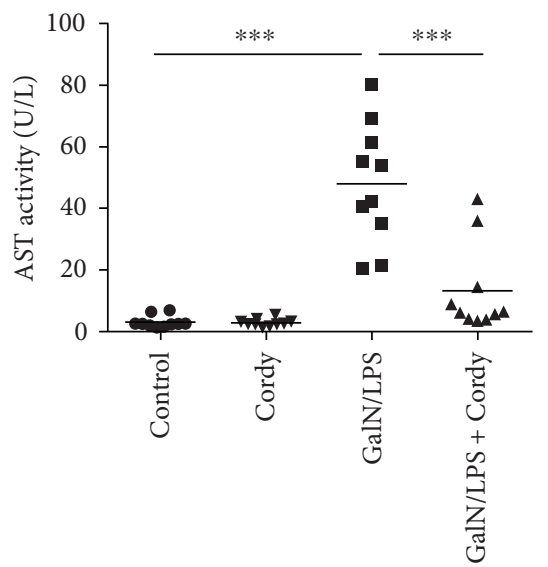

(c)
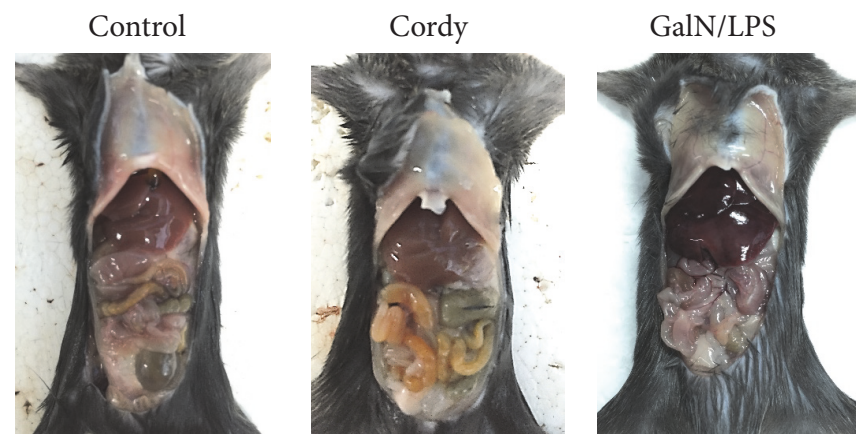

GalN/LPS + Cordy

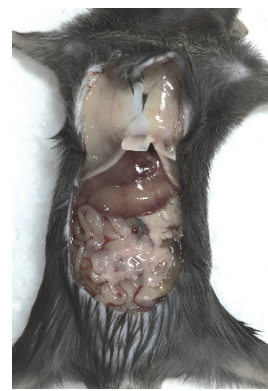

(d)

Figure 1: Continued. 

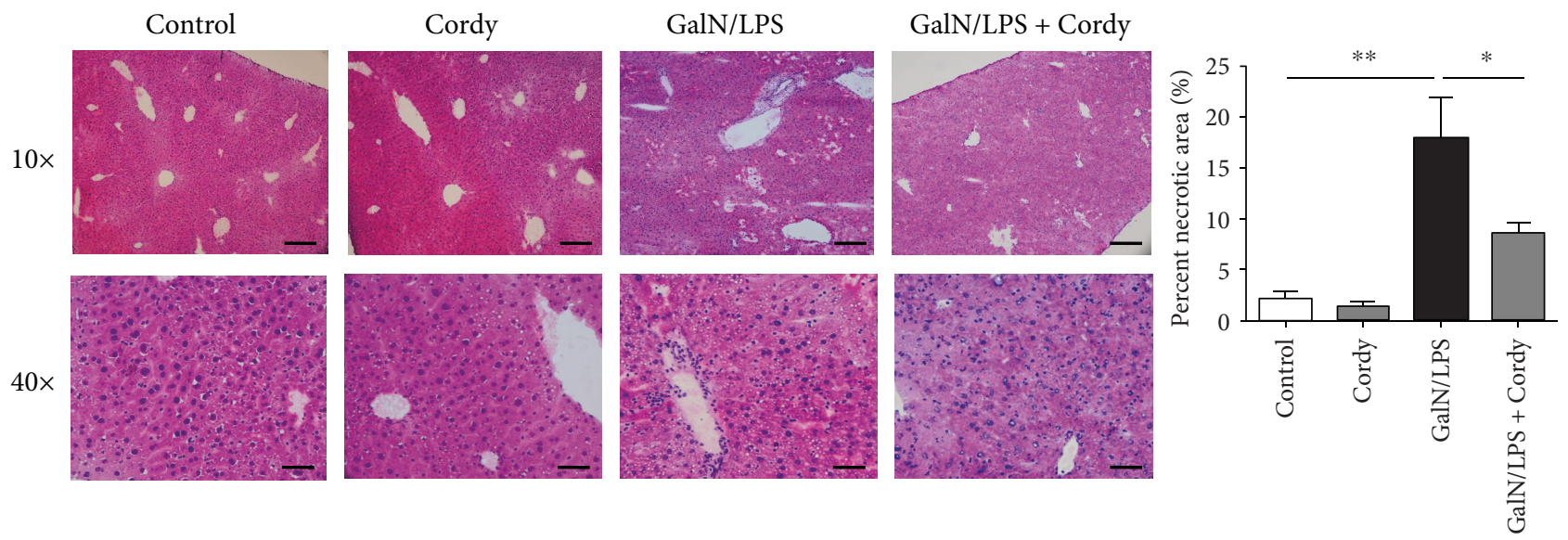

(e)

FIGURE 1: Cordycepin protects against hepatic damage induced by GalN/LPS. C57BL/6J mice were orally administrated with $50 \mathrm{mg} / \mathrm{kg}$ or $200 \mathrm{mg} / \mathrm{kg}$ cordycepin for 3 consecutive weeks, and GalN $(700 \mathrm{mg} / \mathrm{kg}) / \mathrm{LPS}(15 \mu \mathrm{g} / \mathrm{kg})$ was injected at $1 \mathrm{~h}$ before the last gavage. (a) The mortality rates were observed at various time points within $12 \mathrm{~h}$ after injected with a lethal dose of GalN/LPS $(n=10)$. The blood and liver tissues were harvested at $5 \mathrm{~h}$ after GalN/LPS treatment. ( $b$ and $c$ ) Then serum ALT, AST, and HMGB1 levels were assayed ( $n=10$ ). Represent visibly different damaged livers (d) and H\&E staining analysis of liver sections (e) were shown. Upper scale bars: $100 \mu \mathrm{m}$. Under scale bars: $50 \mu \mathrm{m}$. Percent of necrotic area was calculated $(n=5)$. Each value is mean \pm SEM. ${ }^{*} P<0.05,{ }^{* *} P<0.01,{ }^{* * *} P<0.001$.

USA) with a magnification of 400x. At least three tissue sections in each group were analyzed.

\subsection{Quantitative Real-Time Polymerase Chain Reaction} ( $q$ RT-PCR) Analysis. Total RNA was extracted from liver tissues by Trizol Reagent (Sigma-Aldrich, St. Louis, USA). cDNAs were synthesized using reverse transcription kit (Toyobo, Shanghai, China) and then was amplified in polymerase chain reactions (PCRs) with SYBR Green Supermix kit (Toyobo, Shanghai, China). The sequences of primers are as follows: mouse TNF- $\alpha$, forward $5^{\prime}$-GCCTCTTCTCA TTCCTGCTTGT-3', reverse 5'-TTGAGATCCATGCCGTT G-3'; mouse IL- $1 \beta$, forward 5'-TTGACGGACCCCAAAAG AT-3', reverse 5'-GATGATCTGAGTGTGAGGGTCTG-3'; mouse IL-6, forward 5'-GCTACCAAACTGGATATAATC AGGA-3', reverse 5'-CCAGGTAGCTATGGTACTCCAGA A-3'; and mouse GAPDH, forward 5'-GCCTGGAGAAAC CTGCCAAGTAT-3', reverse 5'-GATGCCTGCTTCACCAC CTTC-3'. The reactions were performed on Applied Biosystems Prism 7900HT. GAPDH served as internal normalization control.

2.8. Western Blot Analysis. Proteins were prepared from liver tissues in RIPA lysis buffer with protease inhibitors and phosphatase inhibitors cocktail (Roche, Switzerland). Then SDS-PAGE was conducted, and bands were transferred to PVDF membrane, incubating with primary antibodies against toll-like receptor 4 (TLR4, diluted at 1:1000, catalog number: ab45104, Abcam, Cambridge, UK), microtubuleassociated protein light chain 3 (LC3, diluted at 1:1000, catalog number: ab48394, Abcam, Cambridge, UK), p62 (diluted at 1:1000, catalog number: ab56416, Abcam, Cambridge, UK), phosphor-IKB (diluted at $1: 1000$, catalog number: ab12135, Abcam, Cambridge, UK), I $\kappa \mathrm{B}$ (diluted at $1: 1000$, catalog number: 4814, Cell Signaling Technology, Boston, USA), caspase-3, caspase-8, caspase-9, and poly-
(ADP-ribose) polymerase (PARP) (diluted at $1: 1000$, catalog numbers: 9665, 4790, 9508, and 9532, Cell Signaling Technology, Boston, USA). Corresponding secondary antibodies (diluted at 1:5000, catalog numbers: ZDR-5306 or ZDR5307, Sino Biological, Beijing, China) were added, and bands were visualized with enhanced chemiluminescence reagents (Thermo Fisher Scientific, Waltham, USA). Signals were normalized to that of $\beta$-actin (diluted at $1: 5000$, catalog number: 4967, Cell Signaling Technology, Boston, USA). Five replicates were used for statistical analysis.

2.9. Electron Microscopic (EM) Analysis. Liver tissues were fixed in $2.5 \%$ glutaraldehyde followed by $1 \% \mathrm{OsO}_{4}$. After dehydration, thin sections were stained with uranyl acetate and lead citrate. Images were acquired by New Bio-TEM H-7500 (Hitachi, Tokyo, Japan).

2.10. Data Analysis. Survival data were analyzed by the Kaplan-Meier curve and log-rank test. All other data were analyzed by one-way analysis of variance (ANOVA), and the Bonferroni test was used for post hoc comparisons. The differences between each group were considered statistically significant at a $p$ value $<0.05$. The results are presented as mean \pm SEM.

\section{Results}

3.1. Pretreatment of Cordycepin Attenuated Acute Hepatic Damage Induced by GalN/LPS. In our study, all mice suffered severe liver injury and died within $12 \mathrm{~h}$ after injection with $700 \mathrm{mg} / \mathrm{kg}$ GalN plus $15 \mu \mathrm{g} / \mathrm{kg}$ LPS. Compared with mice only treated with GalN/LPS, there was no markedly protective effect in mice administrated with $50 \mathrm{mg} / \mathrm{kg}$ cordycepin, but with a dose of $200 \mathrm{mg} / \mathrm{kg}$ cordycepin, the survival time significantly prolonged and the mortality markedly decreased approximately by $30 \%$ (Figure 1(a)). 


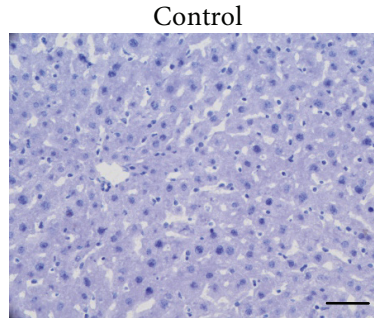

GalN/LPS

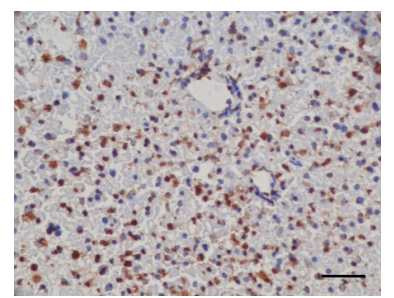

Cordy

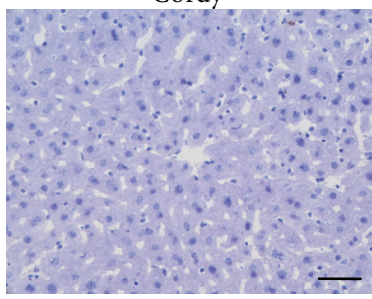

GalN/LPS + Cordy

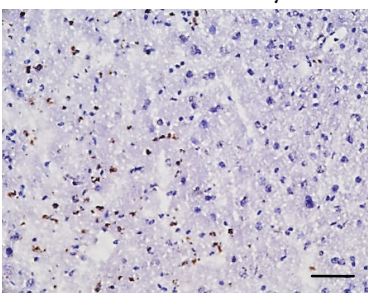

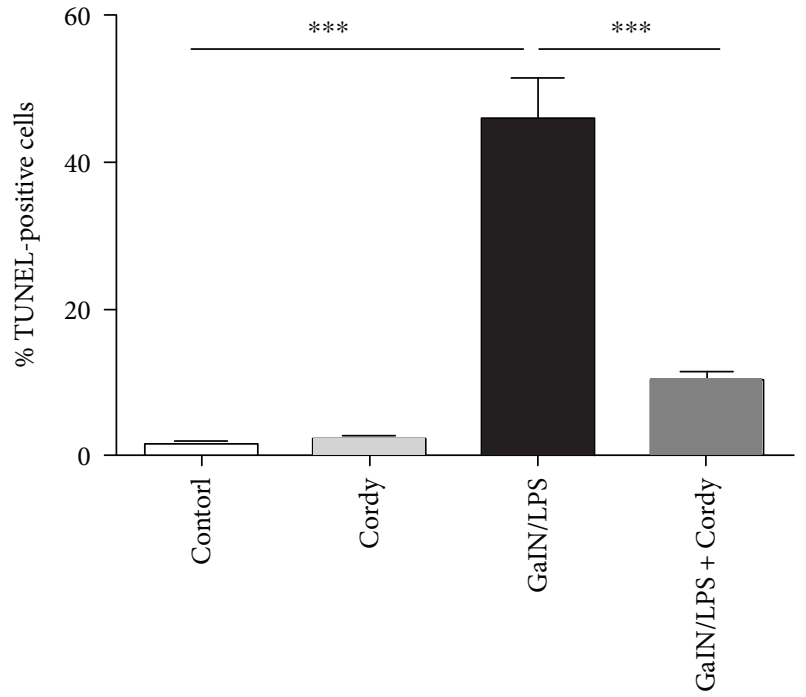

(a)
$116 \mathrm{kDa}$ $89 \mathrm{kDa}$

$35 \mathrm{kDa}$

$19 \mathrm{kDa}$ $17 \mathrm{kDa}$

Cleaved caspase-3

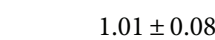

$1.02 \pm 0.05$

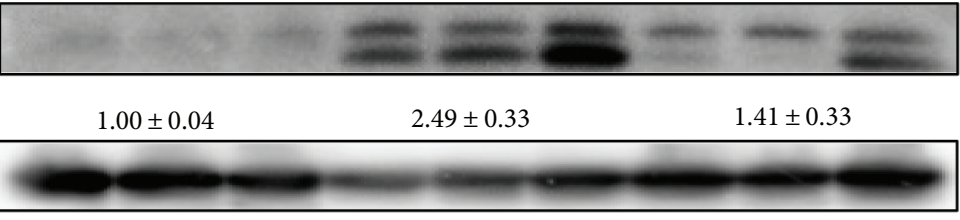

$57 \mathrm{kDa}$

Procaspase- 8

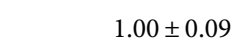

$0.64 \pm 0.06$

$0.82 \pm 0.08$

Cleaved caspase- 8

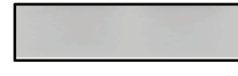

$1.00 \pm 0.08$

$1.72 \pm 0.35$

$1.20 \pm 0.18$

Procaspase- 9

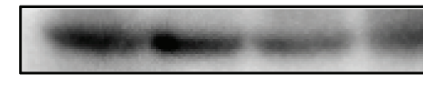

$1.00 \pm 0.09$

$0.45 \pm 0.12$

$1.13 \pm 0.03$

Cleaved caspase- 9

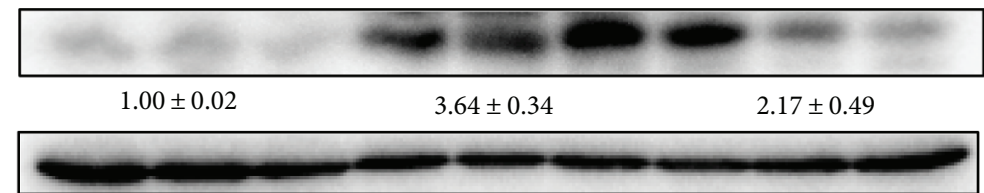

$37 \mathrm{kDa}$

$45 \mathrm{kDa}$

(b)

FIGURE 2: Cordycepin reduces hepatocyte apoptosis caused by GalN/LPS injection. Murine liver tissues were collected at $5 \mathrm{~h}$ after injected with GalN $(700 \mathrm{mg} / \mathrm{kg}) / \mathrm{LPS}(15 \mu \mathrm{g} / \mathrm{kg})$. (a) Hepatocyte apoptosis were measured by TUNEL staining, and the number of apoptotic cells were counted $(n=3)$. Scale bars: $50 \mu \mathrm{m}$. (b) The level of cleaved caspase-3, caspase- 8 , and caspase- 9 and PARP protein expressions were measured as indicated $(n=5)$. Each value is mean \pm SEM. ${ }^{*} P<0.05,{ }^{* *} P<0.01,{ }^{* * *} P<0.001$.

Meanwhile, the levels of plasma ALT, AST, and HMBG1, which indicate hepatic function and hepatocyte necrosis, were also determined at $5 \mathrm{~h}$ after GalN/LPS injection. Apparent increases in serum ALT, AST, and HMBG1 were observed in mice injected with GalN/LPS alone compared with control group, whereas cordycepin treatment at $200 \mathrm{mg} / \mathrm{kg}$ suppressed their growth (Figures 1(b) and 1(c)). From both macroscopic examination and histopathological inspection, GalN/LPS treatment caused many necrotic hepatocytes in centrilobular region, massive immune cell 

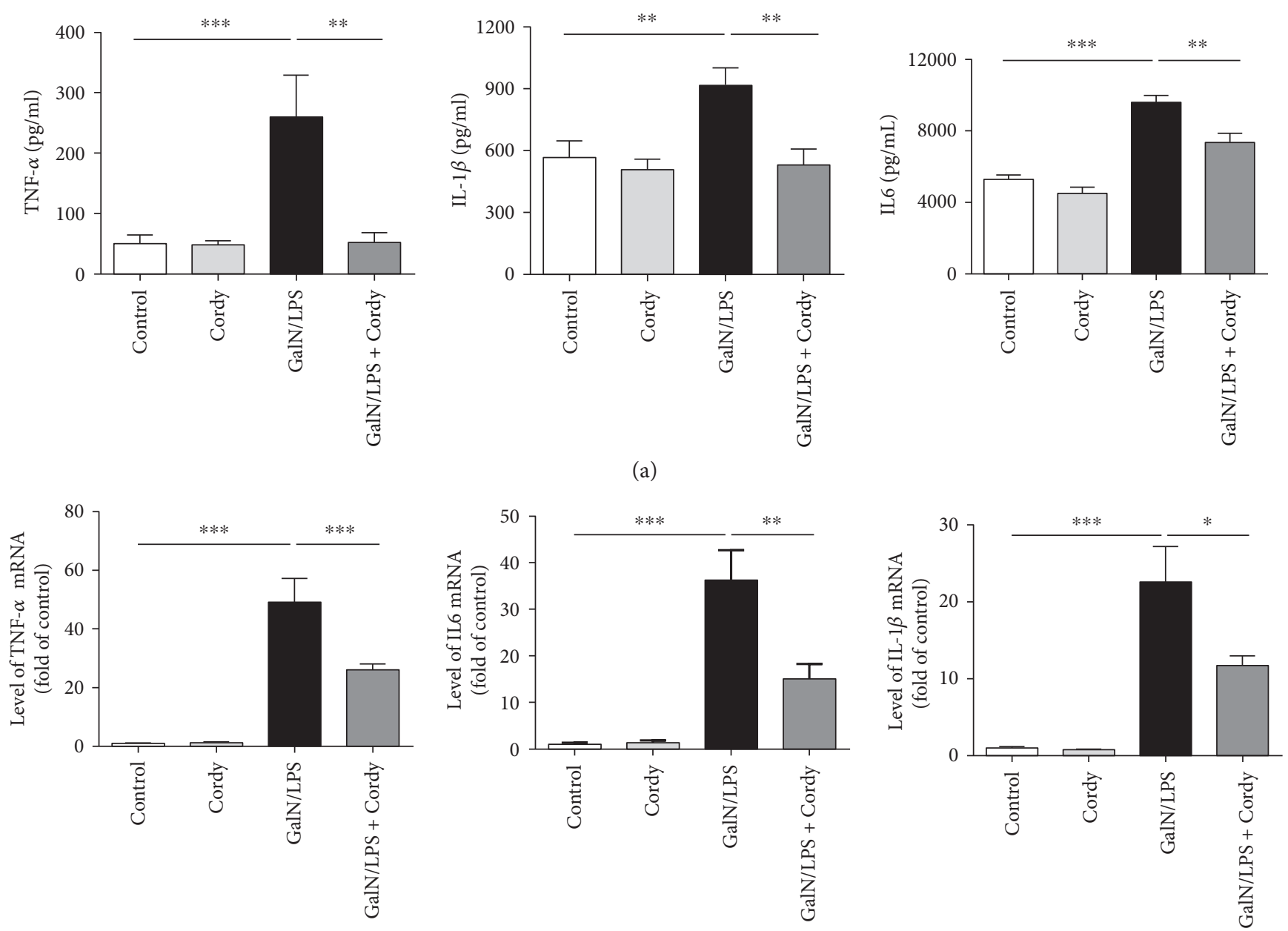

(b)

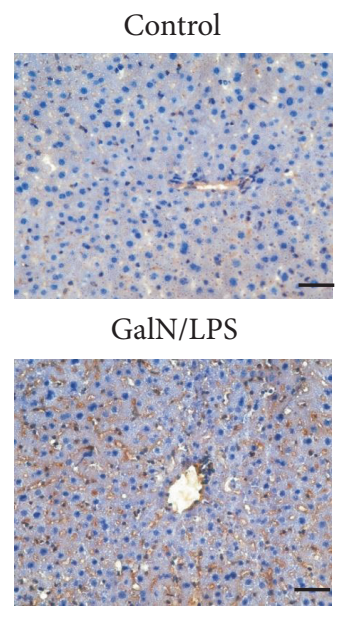

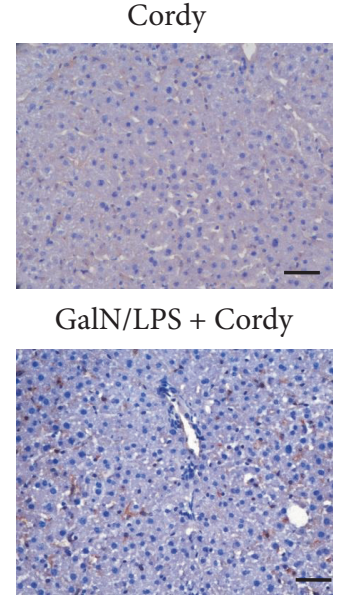

(c)

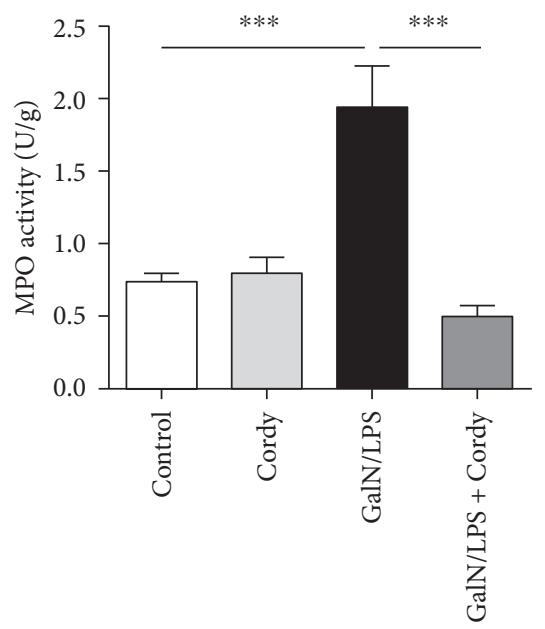

(d)

Figure 3: Continued. 


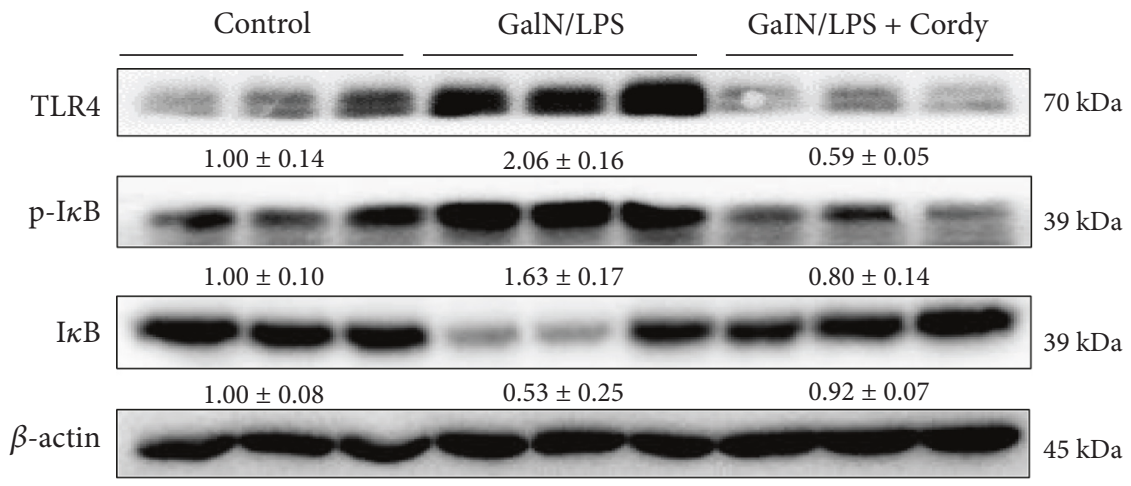

(e)

FIGURE 3: Cordycepin suppresses inflammatory cytokine production and attenuates TLR4 and NF $\kappa$ B signal activation. Serum samples and liver tissues were harvested at $5 \mathrm{~h}$ after injected with GalN/LPS to mice. (a) Serum TNF- $\alpha$, IL-1 $\beta$, and IL-6 levels were assayed using ELISA method $(n=8)$. (b) Hepatic TNF- $\alpha$, IL-1 $\beta$, and IL- 6 mRNA expressions were determined by qRT-PCR $(n=5)$. (c) F4/80 staining of liver sections were conducted to indicate the filtration of macrophages. Scale bars: $50 \mu \mathrm{m}$. (d) Hepatic MPO activity was measured according to manufacturer's instruction $(n=8)$. (e) Hepatic expression of TLR4, I $\kappa \mathrm{B}$, and p-I $\kappa \mathrm{B}$ was analyzed by western blotting $(n=5)$. Each value is mean \pm SEM. ${ }^{*} P<0.05,{ }^{* *} P<0.01,{ }^{* * *} P<0.001$.

immigration to sinusoids, and hemorrhage, which were tremendously ameliorated by cordycepin administration at a dose of $200 \mathrm{mg} / \mathrm{kg}$ (Figures 1(d) and 1(e)).

As shown in Figure 2(a) using TUNEL method, the number of apoptotic cells greatly increased due to GalN/LPS injection $(46.2 \pm 11.8$ per 100 nuclei), whereas pretreatment of $200 \mathrm{mg} / \mathrm{kg}$ cordycepin prevented hepatocyte apoptosis (10.4 \pm 2.6 per 100 nuclei). In accordance with TUNEL data, the concentrations of cleaved caspase- 8 and caspase-9, one mediated death receptor pathway and the other mediated mitochondrial intrinsic pathway, were upregulated; nevertheless, the expressions of full length ones were downregulated in GalN/LPS-treated group. Similar circumstances were present in both caspase- 3 and PARP, which are effectors of apoptotic pathway; however, cordycepin $(200 \mathrm{mg} / \mathrm{kg})$ administration reversed these changes (Figure 2(b)). In cordycepin-alone treated group, no abnormal alterations were found in these indexes described above when compared with normal mice.

\subsection{Cordycepin Inhibited Hepatic Inflammatory Cytokine} Production and Inflammatory Cell Infiltration Induced by GalN/LPS. Since TNF- $\alpha$ and other inflammatory cytokines such as IL6 and IL-1 $\beta$ are important contributors to liver damage induced by GalN/LPS, we then assayed circulating TNF- $\alpha$, IL6, and IL- $1 \beta$ levels at $5 \mathrm{~h}$ after GalN/LPS injection. As expected, GalN/LPS induced remarkable elevation of serum TNF- $\alpha$, IL6, and IL- $1 \beta$ levels in contrast to those in the control groups, but pretreatment with $200 \mathrm{mg} / \mathrm{kg}$ cordycepin tremendously inhibited their production (Figure 3(a)). Similar results were obtained when measuring the mRNA expression of TNF- $\alpha$, IL6, and IL- $1 \beta$ in liver tissues, demonstrating that cordycepin can inhibit GalN/LPS-induced inflammatory cytokine production through suppressing mRNA transcription (Figure 3(b)).

As Kupffer cells (hepatic macrophages) are the primary sources for these inflammatory cytokines when suffered with
LPS challenge, we next conducted immunohistochemistry experiments with F4/80 antibody, which is a wellestablished marker of hepatic macrophages. As shown in Figure 3(c), there were massive inflammatory cell infiltration into liver tissues after GalN/LPS challenge while cordycepin $(200 \mathrm{mg} / \mathrm{kg})$ attenuated the increase of local macrophages. Equally, GalN/LPS induced an elevation of hepatic MPO activity, another sign for macrophage or neutrophil infiltration, which was also prevented by $200 \mathrm{mg} / \mathrm{kg}$ cordycepin (Figure 3(d)). Identically, the normal animals received with cordycepin had no significant changes when compared with the control group. Therefore, cordycepin was believed to exert hepatoprotective effect in animal model treated with GalN/LPS partly due to its inhibition of inflammatory response.

3.3. Cordycepin Blocked Hepatic TLR4 Expression and NF- $\kappa B$ Activation Induced by GalN/LPS. It has been suggested that TLR4 and its downstream NFkB signal pathway are mainly implicated in LPS-triggered macrophage inflammatory cytokine release [19]. $\mathrm{I} \kappa \mathrm{B}$ was confirmed to be a critical inhibitor for $\mathrm{NF} \kappa \mathrm{B}$, and phosphorylation in Ser32 accelerated its degradation [20]. Therefore, the levels of $\mathrm{I} \kappa \mathrm{B}$ and phosphor$\mathrm{I} \kappa \mathrm{B}$ reflect the activity of $\mathrm{NF} \kappa \mathrm{B}$ indirectly. Then we test whether cordycepin could affect TLR $4, \mathrm{I} \kappa \mathrm{B}$, and phosphor$\mathrm{I} \kappa \mathrm{B}$ expression in vivo. The results of western blotting indicated that GalN/LPS-induced elevated expression of TLR4 and phosphorylated $\mathrm{I} \kappa \mathrm{B}$ in hepatocytes were attenuated by pretreatment with $200 \mathrm{mg} / \mathrm{kg}$ cordycepin. Similarly, the reduced expression of hepatic $\mathrm{I} \kappa \mathrm{B}$ by GalN/LPS injection was also reversed by $200 \mathrm{mg} / \mathrm{kg}$ cordycepin (Figure 3(e)), implying that cordycepin might exert its anti-inflammatory effect via decreasing TLR4 expression and inhibiting NF $\kappa \mathrm{B}$ activation in GalN/LPS-induced acute liver injury.

3.4. Cordycepin Inhibited Oxidative Stress Induced by GalN/ LPS. During the initiation of liver damage, neutrophils or 
macrophages release large quantities of ROS by the phagocytic isoform of NOX complex [21]. To ascertain whether oxidative stress is involved in cordycepin's hepatoprotection against GalN/LPS-induced injury, the contents of lipid peroxidation product MDA, reductive product GSH, and ROS were determined. We observed significant accumulations of intracellular MDA and ROS as well as a reduction of GSH concentration in liver tissues of GalN/LPS-treated mice, which was reversed by the pretreatment with $200 \mathrm{mg} / \mathrm{kg}$ cordycepin (Figures 4(a) and 4(b)). Besides, a great impairment of enhanced NOX activity induced by GalN/LPS was found in the livers of mice pretreated with $200 \mathrm{mg} / \mathrm{kg}$ cordycepin (Figure 4(c)). Together, these data supported that cordycepin may improve oxidative stress in damaged liver, another potential explanation for its protection against GalN/LPSinduced hepatic injury.

3.5. Cordycepin Prevented Excessive Autophagy Induced by GalN/LPS. Mitochondria autophagy (also called mitophagy) is usually induced to self-remove dysfunctional mitochondria when suffered stress. However, excessive levels of autophagy are frequently associated with cell death [22]. From the results of electron macroscopy (Figure 5(a)), we could find electron-scarce cytoplasm and abundant late autophagic vacuoles (autolysosomes), characterized by single limiting membrane surrounding the electron-dense mitochondria debris, in hepatocytes from mice by GalN/LPS administration. Pretreatment with $200 \mathrm{mg} / \mathrm{kg}$ cordycepin significantly reduced the number of autolysosomes. LC3, a mammalian homolog of yeast Atg8, is known as a marker for macroautophagy. LC3-I is localized in cytoplasm while LC3-II (the phosphatidylethanolamine-conjugated form of LC3-I) exists in the membranes of the autophagosome. After fusion with the lysosome, LC3-II is degraded by lysosomal enzymes. Therefore, chronic activation of autophagic flux contributes to decreased expression of total LC3 [23]. Additionally, p62 (SQSTM1/sequestome 1), the autophagy substrate, is selectively degraded in autophagic flux [24]. Thus, p62 level is basically inversely correlated with autophagic degradation [25]. The western blot data showed decreased concentrations of p62 and total LC3 in liver tissues from model group whereas $200 \mathrm{mg} / \mathrm{kg}$ cordycepin treatment increased their expression (Figure 5(b)), demonstrating that cordycepin can prevent GalN/LPS-induced excessive autophagy.

\section{Discussion}

Mice injected with lethal dose of GalN/LPS to induce acute liver injury aims to replicate viral hepatitis-induced fulminant hepatic failure in clinic. Until now, it has become one of the most prevalent experimental murine models to investigate the potential therapeutic tools of fulminant hepatic failure. In the current study, we investigate the protective effects of cordycepin on acute liver injury induced by GalN/ LPS. Specifically, pretreatment with cordycepin could raise the survival rate, improve liver function, decrease hepatocyte necrosis and apoptosis, and attenuate excessive autophagy, suggesting cordycepin may be an attractive therapeutic agent for viral hepatitis-induced fulminant hepatic failure.

Cordycepin, one of the primary bioactive components of Cordyceps militaris, has been reported to possess with antiinflammatory and antioxidant activities. In LPS-stimulated macrophages and BV2 microglia, cordycepin significantly reduced the production of proinflammatory cytokines $[12,26]$. In aged animal models, cordycepin treatment decreased oxidative damage and boosted the differentiation of bone marrow mesenchymal stem cells [27]. Emerging researches showed that abundant macrophage infiltration into the liver, producing excessive proinflammatory cytokines especially TNF- $\alpha$, are the central steps to trigger hepatocyte apoptosis in GalN/LPS-induced acute liver injury [28]. For example, TNF- $\alpha$ or TNF- $\alpha$ receptor-deficient mice was able to resist the challenge of GalN/LPS [29]. In later stage, attracted neutrophils and monocytes by inflammatory cytokines and chemokines generate massive ROS and protease, contributing to hepatocyte necrosis, which is characterized by increased plasma HMGB1 released from dysfunctional tissues $[28,30]$. Therefore, we speculated that administration with cordycepin might be profitable for acute liver injury caused by GalN/LPS injection. Indeed, our present results showed that cordycepin effectively antagonized GalN/LPSinduced elevated serum TNF- $\alpha$ levels, increased concentrations of hepatic ROS and oxidative product MDA, and decreased reductive product GSH, leading to alleviation of GalN/LPS causing impaired liver function (elevated ALT and AST) and massive hepatocyte apoptosis and necrosis, which is probably the reason why cordycepin could lower the high mortality rate induced by GalN/LPS.

To further explore the underlying mechanism by which cordycepin exerted anti-inflammatory effect, we assessed the expression of hepatic TNF- $\alpha$ mRNA, the activity of its leading transcriptional factor $\mathrm{NF} \kappa \mathrm{B}$, and the level of TLR4 protein. The canonical $\mathrm{NF} \kappa \mathrm{B}$ signaling pathway is triggered once LPS interacts with its receptor TLR4 on the surface of hepatic macrophage [31]. Subsequently, $I \kappa B$, known as the inhibitor of $\mathrm{NF} \kappa \mathrm{B}$, is phosphorylated by upstream kinase IKK and sequential ubiquitinated which leads to its degradation. As expected, the current data demonstrated that cordycepin prevented the elevated expression of TLR4, depressed the phosphorylation of $\mathrm{I} \kappa \mathrm{B}$, and thus blocked GalN/LPSinduced $\mathrm{NF} \kappa \mathrm{B}$ activation in the liver. The following elevated TNF- $\alpha$ mRNA level was also inhibited by administration with cordycepin, which may explain why there was lower concentration of plasma TNF- $\alpha$ compared to GalN/LPS treatment alone.

Enhanced MPO activity is the specific warning signal for neutrophil and macrophage infiltration, which is the dominant resource for ROS production in early stage [32]. Our data declared that cordycepin strikingly downregulated MPO activity, manifesting fewer hepatic neutrophils and macrophages, consistent with the result of F4/80 immunohistochemical stain, another biomarker for murine macrophage population [33]. Activated neutrophils and macrophages produce massive ROS, which will further incur hepatocyte necrosis. There are two main resources for ROS, one from NOX complex, which generates reactive oxygen 

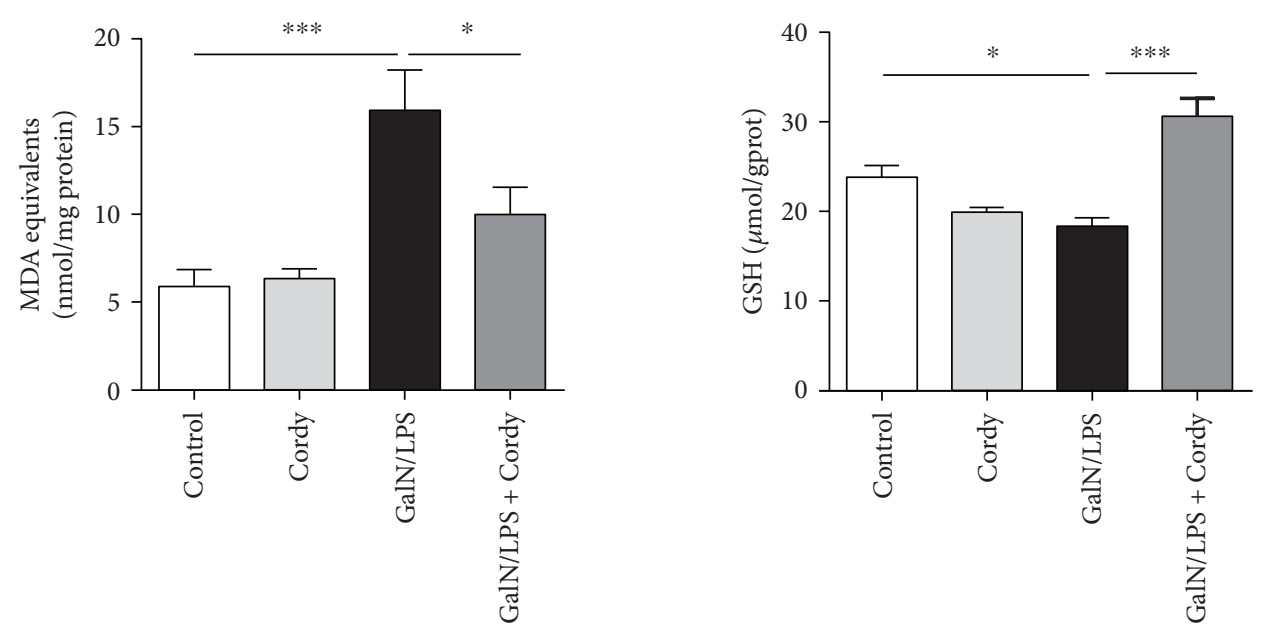

(a)

Control

Cordy

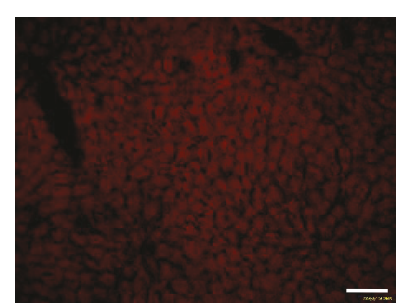

GalN/LPS

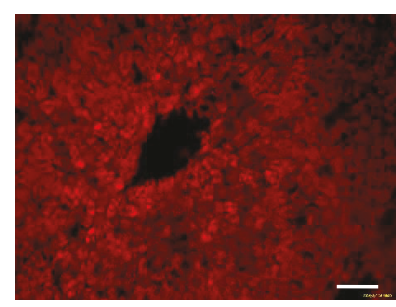

GalN/LPS + Cordy

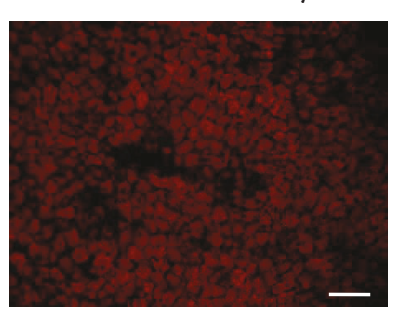

(b)

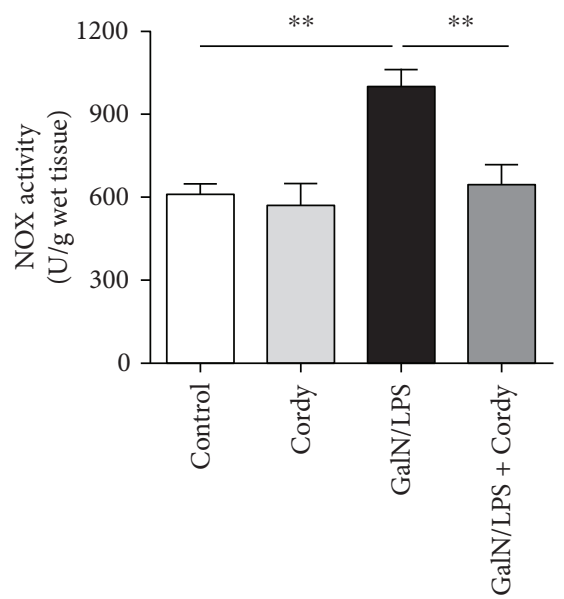

(c)

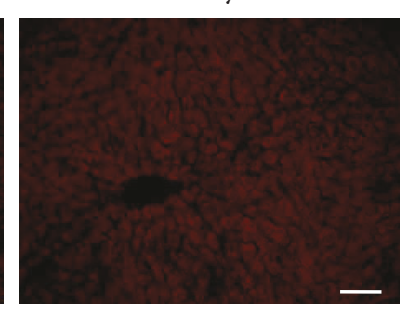

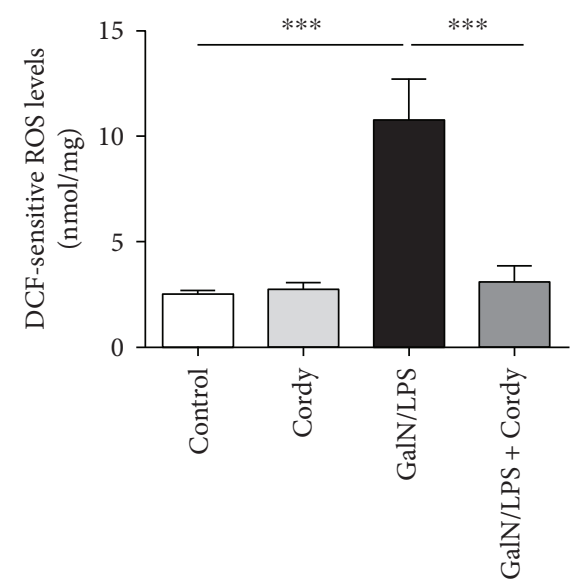

Figure 4: Cordycepin prevents oxidative stress induced by GalN/LPS. Murine liver tissues were collected at $5 \mathrm{~h}$ after injected with GalN $(700 \mathrm{mg} / \mathrm{kg}) / \mathrm{LPS}(15 \mu \mathrm{g} / \mathrm{kg})$. (a) Hepatic MDA and GSH levels were determined using commercial kits $(n=8)$. (b) The ROS levels in the liver tissues were visualized by DHE staining and measured by DCF assay $(n=8)$. Scale bars: $50 \mu \mathrm{m}$. (c) Hepatic NOX activity was determined using NADH as substrates $(n=5)$. Each value is mean \pm SEM. ${ }^{*} P<0.05,{ }^{* *} P<0.01,{ }^{* * *} P<0.001$. 

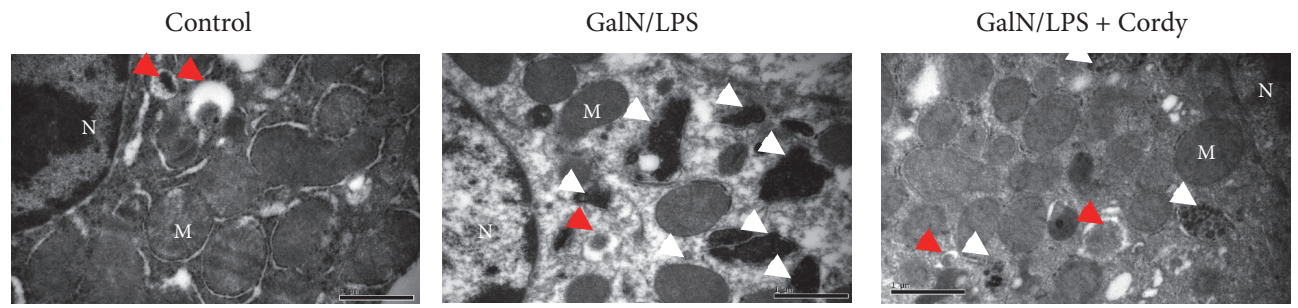

(a)

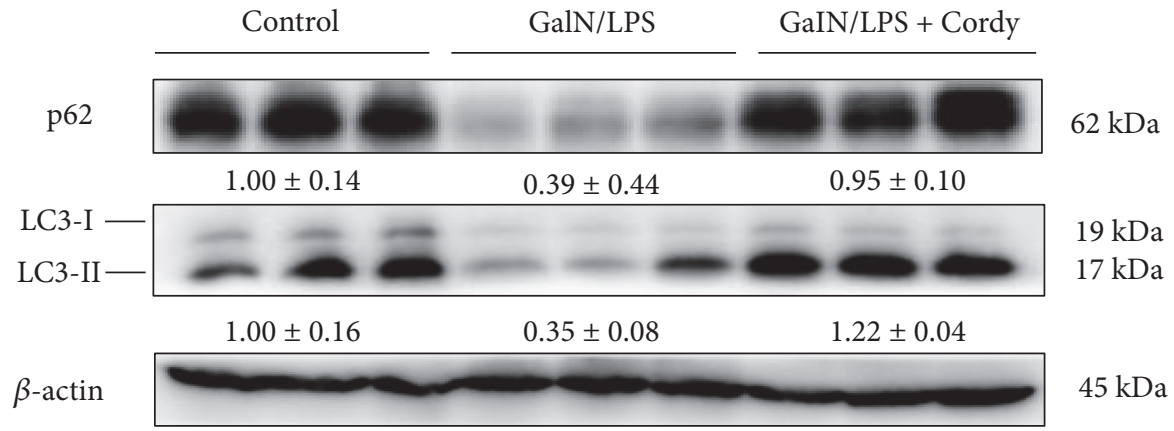

(b)

FIGURE 5: Cordycepin blocks excessive autophagy. Murine liver tissues were collected at $5 \mathrm{~h}$ after injected with GalN (700 mg/kg)/LPS $(15 \mu \mathrm{g} / \mathrm{kg})$. (a) Electron microscope analysis of liver tissues. Red arrows denote autophagosomes. White arrows denote autolysosomes. $\mathrm{N}$ : nucleus; M: mitochondria. Bars: $1 \mu \mathrm{m}$. (b) Immunoblot analysis of LC3 and p62 protein expressions $(n=5)$. Each value is mean \pm SEM.

using $\mathrm{NAD}(\mathrm{P}) \mathrm{H}$ as substrates. In the current study, reduced NOX activity was found in livers from mice pretreated with cordycepin when compared with mice injected with GalN/ LPS, suggesting that cordycepin may improve oxidative stress via inhibiting NOX activity. Similar results were obtained in albumin-induced epithelial-mesenchymal transition of renal tubular cells [34]. However, whether there is direct suppressing effect on NOX complex by cordycepin remains unknown and further experiments are required.

Another principal source for ROS is the leakage from electron transfer chain in the mitochondria, especially the damaged ones. Massive superoxide release lead to sustained activation of c-Jun N-terminal kinase (JNK) and finally cause necrotic cell death [35]. Meanwhile, mitochondria autophagy (also called mitophagy) is induced to selectively eliminate dysfunctional ones, which seems to be protective. However, bulk mitophagy was also observed accompanying with great numbers of dying cells. It was reported that GalN/LPS could induce autophagic flux in dysfunctional liver [36], and we also found abundant autolysosomes present in liver tissues of mice suffered with GalN/LPS challenge. Pretreatment with cordycepin reduced excessive levels of autolysosomes and inhibited autophagic activity. But whether cordycepin is an inhibitor of autophagy or decreases autophagic activity indirectly through suppressing oxidative stress remains to be determined.

\section{Conclusion}

Pretreatment with cordycepin is beneficial for acute liver injury caused by GalN/LPS injection for its antiinflammation and antioxidation property. The underlying mechanism might be associated with the decreased expression of TLR4, the inhibition of $\mathrm{NF} \kappa \mathrm{B}$ signaling pathway, and the following depressed generation of TNF- $\alpha$, contributing to reduced hepatocyte apoptosis. Decreased NOX activity and suppressed production of ROS help alleviate oxidative damage and reduce hepatocyte necrosis. Besides, cordycepin is also shown to prevent excessive autophagic activity induced by GalN/LPS.

\section{Conflicts of Interest}

All authors declare no conflict of interest.

\section{Acknowledgments}

This study was supported by grants from the Science Innovation Project of the Chinese Academy of Agricultural Sciences (no. 125161015000150013), CAMS Initiative for Innovative Medicine (no. CAMS-I2M-1-009), and National Natural Science Foundation of China (nos. 81302827, 81273514, 91539126, and 81603172).

\section{References}

[1] Q. Xiong, K. Hase, Y. Tezuka, T. Namba, and S. Kadota, "Acteoside inhibits apoptosis in D-galactosamine and lipopolysaccharide-induced liver injury," Life Sciences, vol. 65, no. 4, pp. 421-430, 1999.

[2] L. Ma, X. Gong, G. Kuang, R. Jiang, R. Chen, and J. Wan, "Sesamin ameliorates lipopolysaccharide/d-galactosamineinduced fulminant hepatic failure by suppression of Toll-like receptor 4 signaling in mice," Biochemical and Biophysical Research Communications, vol. 461, no. 2, pp. 230-236, 2015. 
[3] H. Malhi, G. J. Gores, and J. J. Lemasters, "Apoptosis and necrosis in the liver: a tale of two deaths?" Hepatology, vol. 43, 2 Supplement 1, pp. S31-S44, 2006.

[4] H. Ohira, K. Abe, J. Yokokawa et al., "Adhesion molecules and CXC chemokines in endotoxin-induced liver injury," Fukushima Journal of Medical Science, vol. 49, no. 1, pp. 1-13, 2003.

[5] D. L. Panicali and C. N. Nair, "Effect of cordycepin triphosphate on in vitro RNA synthesis by picornavirus polymerase complexes," Journal of Virology, vol. 25, no. 1, pp. 124-128, 1978.

[6] A. M. Sugar and R. P. McCaffrey, "Antifungal activity of 3'-deoxyadenosine (cordycepin)," Antimicrobial Agents and Chemotherapy, vol. 42, no. 6, pp. 1424-1427, 1998.

[7] Y. Koc, A. G. Urbano, E. B. Sweeney, and R. McCaffrey, "Induction of apoptosis by cordycepin in ADA-inhibited TdT-positive leukemia cells," Leukemia, vol. 10, no. 6, pp. 1019-1024, 1996.

[8] Y. Liao, J. Ling, G. Zhang et al., "Cordycepin induces cell cycle arrest and apoptosis by inducing DNA damage and upregulation of p53 in Leukemia cells," Cell Cycle, vol. 14, no. 5, pp. 761-771, 2015.

[9] K. J. Won, S. C. Lee, C. K. Lee et al., "Cordycepin attenuates neointimal formation by inhibiting reactive oxygen speciesmediated responses in vascular smooth muscle cells in rats," Journal of Pharmacological Sciences, vol. 109, no. 3, pp. 403-412, 2009.

[10] S. Takahashi, M. Tamai, S. Nakajima et al., "Blockade of adipocyte differentiation by cordycepin," British Journal of Pharmacology, vol. 167, no. 3, pp. 561-575, 2012.

[11] H. J. Cho, J. Y. Cho, M. H. Rhee, and H. J. Park, "Cordycepin (3'-deoxyadenosine) inhibits human platelet aggregation in a cyclic AMP- and cyclic GMP-dependent manner," European Journal of Pharmacology, vol. 558, no. 1-3, pp. 43-51, 2007.

[12] H. G. Kim, B. Shrestha, S. Y. Lim et al., "Cordycepin inhibits lipopolysaccharide-induced inflammation by the suppression of NF-kappaB through Akt and p38 inhibition in RAW 264.7 macrophage cells," European Journal of Pharmacology, vol. 545, no. 2-3, pp. 192-199, 2006.

[13] S. Shin, S. Moon, Y. Park et al., "Role of cordycepin and adenosine on the phenotypic switch of macrophages via induced anti-inflammatory cytokines," Immune Network, vol. 9, no. 6, pp. 255-264, 2009.

[14] D. W. Zhang, Z. L. Wang, W. Qi, W. Lei, and G. Y. Zhao, "Cordycepin (3'-deoxyadenosine) down-regulates the proinflammatory cytokines in inflammation-induced osteoporosis model," Inflammation, vol. 37, no. 4, pp. 1044-1049, 2014.

[15] J. Y. Cha, H. Y. Ahn, Y. S. Cho, and J. Y. Je, "Protective effect of cordycepin-enriched Cordyceps militaris on alcoholic hepatotoxicity in Sprague-Dawley rats," Food and Chemical Toxicology, vol. 60, pp. 52-57, 2013.

[16] Y. Sun, Y. H. Wang, K. Qu, and H. B. Zhu, "Beneficial effects of cordycepin on metabolic profiles of liver and plasma from hyperlipidemic hamsters," Journal of Asian Natural Products Research, vol. 13, no. 6, pp. 534-546, 2011.

[17] C. K. Ayata, S. C. Ganal, B. Hockenjos et al., "Purinergic $\mathrm{P} 2 \mathrm{Y}(2)$ receptors promote neutrophil infiltration and hepatocyte death in mice with acute liver injury," Gastroenterology, vol. 143, no. 6, pp. 1620-1629, 2012, e1624.

[18] Z. Lin, F. Wu, S. Lin et al., "Adiponectin protects against acetaminophen-induced mitochondrial dysfunction and acute liver injury by promoting autophagy in mice," Journal of Hepatology, vol. 61, no. 4, pp. 825-831, 2014.

[19] H. Oura and Y. Ichikawa, "The classic text Sugiyama Sindenryu, as re-compiled by Masanaga Wada," Nihon Ishigaku Zasshi, vol. 55, no. 3, pp. 329-345, 2009.

[20] Y. Yamamoto and R. B. Gaynor, "IkappaB kinases: key regulators of the NF-kappaB pathway," Trends in Biochemical Sciences, vol. 29, no. 2, pp. 72-79, 2004.

[21] J. Cuschieri and R. V. Maier, "Oxidative stress, lipid rafts, and macrophage reprogramming," Antioxidants \& Redox Signaling, vol. 9, no. 9, pp. 1485-1497, 2007.

[22] G. Kroemer and B. Levine, "Autophagic cell death: the story of a misnomer," Nature Reviews. Molecular Cell Biology, vol. 9, no. 12, pp. 1004-1010, 2008.

[23] N. Mizushima and T. Yoshimori, "How to interpret LC3 immunoblotting," Autophagy, vol. 3, no. 6, pp. 542-545, 2007.

[24] G. Bjorkoy, T. Lamark, A. Brech et al., "p62/SQSTM1 forms protein aggregates degraded by autophagy and has a protective effect on huntingtin-induced cell death," The Journal of Cell Biology, vol. 171, no. 4, pp. 603-614, 2005.

[25] B. J. Bartlett, P. Isakson, J. Lewerenz et al., "p62, Ref(2)P and ubiquitinated proteins are conserved markers of neuronal aging, aggregate formation and progressive autophagic defects," Autophagy, vol. 7, no. 6, pp. 572-583, 2011.

[26] J. W. Jeong, C. Y. Jin, G. Y. Kim et al., "Anti-inflammatory effects of cordycepin via suppression of inflammatory mediators in BV2 microglial cells," International Immunopharmacology, vol. 10, no. 12, pp. 1580-1586, 2010.

[27] F. Wang, P. Yin, Y. Lu et al., "Cordycepin prevents oxidative stress-induced inhibition of osteogenesis," Oncotarget, vol. 6, no. 34, pp. 35496-35508, 2015.

[28] H. W. Zimmermann, C. Trautwein, and F. Tacke, "Functional role of monocytes and macrophages for the inflammatory response in acute liver injury," Frontiers in Physiology, vol. 3, p. 56, 2012.

[29] M. Nowak, G. C. Gaines, J. Rosenberg et al., "LPS-induced liver injury in D-galactosamine-sensitized mice requires secreted TNF-alpha and the TNF-p55 receptor," American Journal of Physiology. Regulatory, Integrative and Comparative Physiology, vol. 278, no. 5, pp. R1202-R1209, 2000.

[30] W. Wang, L. Sun, Y. Deng, and J. Tang, "Synergistic effects of antibodies against high-mobility group box 1 and tumor necrosis factor-alpha antibodies on $\mathrm{D}$-(+)-galactosamine hydrochloride/lipopolysaccharide-induced acute liver failure," The FEBS Journal, vol. 280, no. 6, pp. 1409-1419, 2013.

[31] O. Takeuchi and S. Akira, "Pattern recognition receptors and inflammation," Cell, vol. 140, no. 6, pp. 805-820, 2010.

[32] Z. Wen, Y. Liu, F. Li, and T. Wen, "Low dose of carbon monoxide intraperitoneal injection provides potent protection against GalN/LPS-induced acute liver injury in mice," Journal of Applied Toxicology, vol. 33, no. 12, pp. 1424-1432, 2013.

[33] H. H. Lin, D. E. Faunce, M. Stacey et al., "The macrophage F4/80 receptor is required for the induction of antigenspecific efferent regulatory $\mathrm{T}$ cells in peripheral tolerance," The Journal of Experimental Medicine, vol. 201, no. 10, pp. 1615-1625, 2005.

[34] L. Xiao, Y. Ge, L. Sun et al., "Cordycepin inhibits albumininduced epithelial-mesenchymal transition of renal tubular epithelial cells by reducing reactive oxygen species production," Free Radical Research, vol. 46, no. 2, pp. 174-183, 2012. 
[35] H. Kamata, S. Honda, S. Maeda, L. Chang, H. Hirata, and M. Karin, "Reactive oxygen species promote TNFalpha-induced death and sustained JNK activation by inhibiting MAP kinase phosphatases," Cell, vol. 120, no. 5, pp. 649-661, 2005.

[36] K. Wang, I. Damjanov, and Y. J. Wan, “The protective role of pregnane $\mathrm{X}$ receptor in lipopolysaccharide/D-galactosamineinduced acute liver injury," Laboratory Investigation, vol. 90, no. 2, pp. 257-265, 2010. 


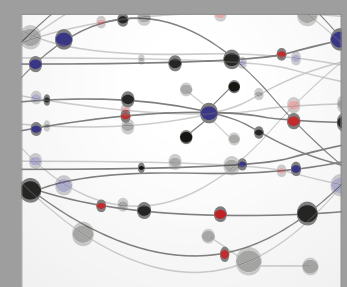

The Scientific World Journal
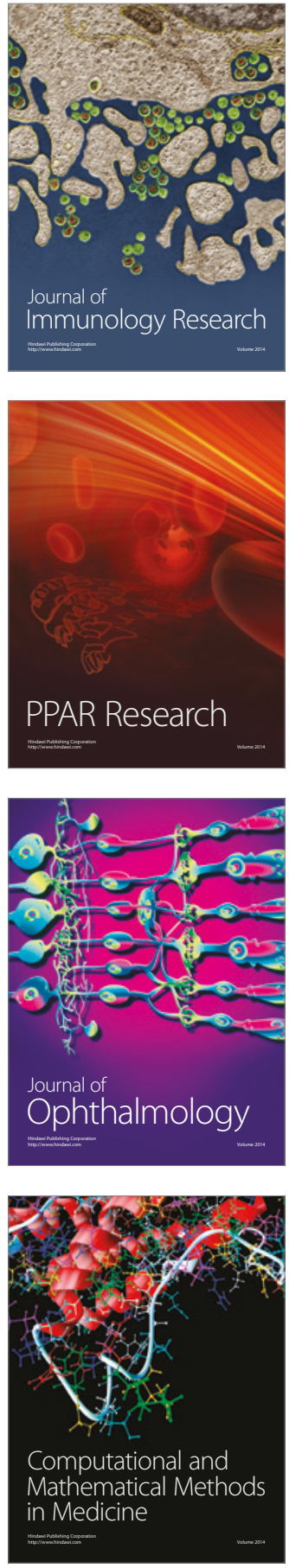

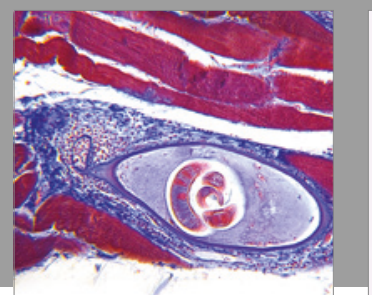

Gastroenterology Research and Practice
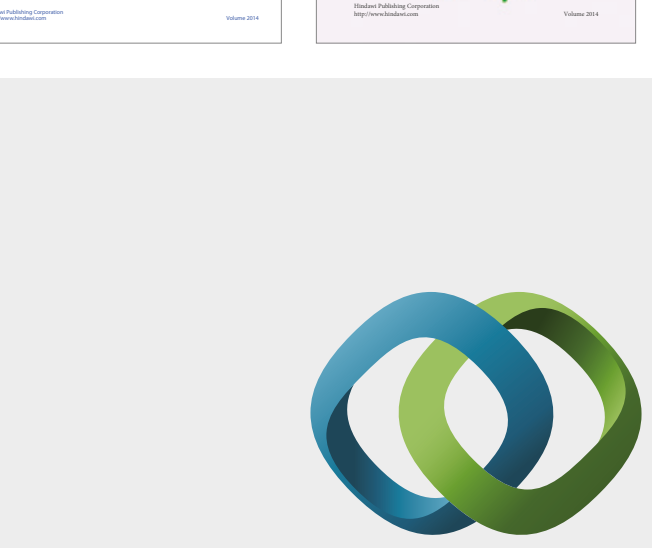

\section{Hindawi}

Submit your manuscripts at

https://www.hindawi.com
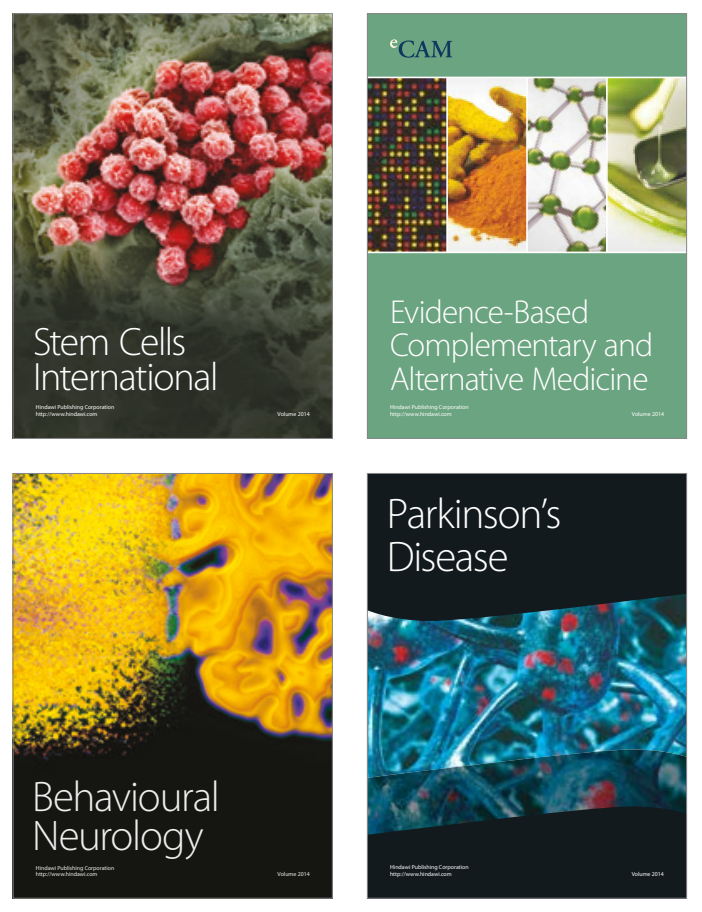
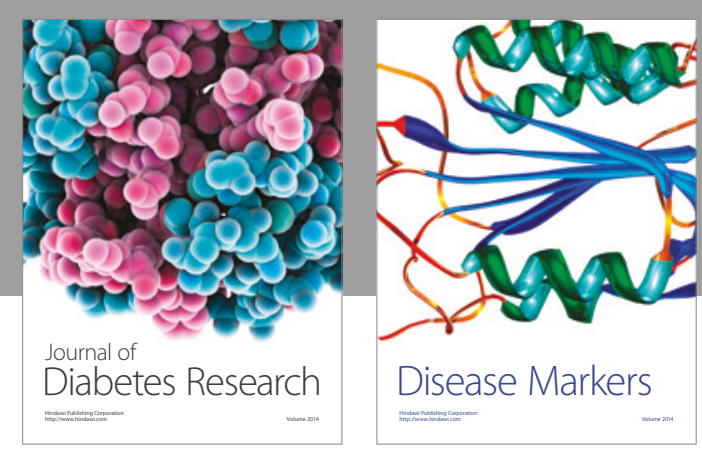

Disease Markers
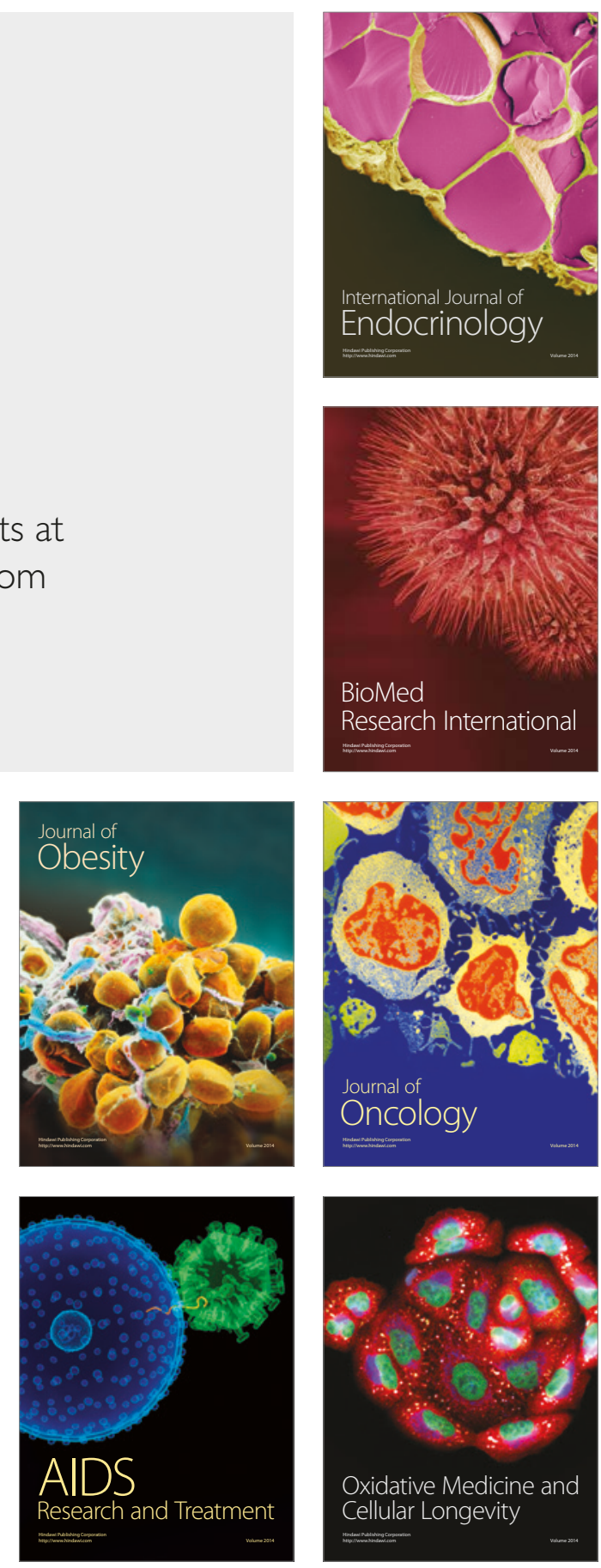